BRAVILLIAN JOURNAL

www.bjournal.com.br
ISSN 0100-879X

Volume 43 (4) 268-380 April 2011

BIOMEDICAL SCIENCES

AND

CLINICAL INVESTIGATION

Braz J Med Biol Res, April 2011, Volume 44(4) 283-290

doi: 10.1590/S0100-879X2011007500022

Overexpression of kermit/dGIPC is associated with lethality in Drosophila melanogaster

G.B. Pereira, V. Valente, M.S. de Queiroz, M.C.B. Vianna and M.L. Paçó-Larson

The Brazilian Journal of Medical and Biological Research is partially financed by

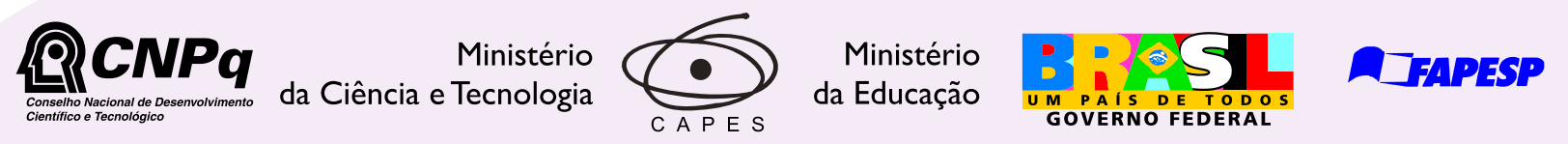

Institutional Sponsors
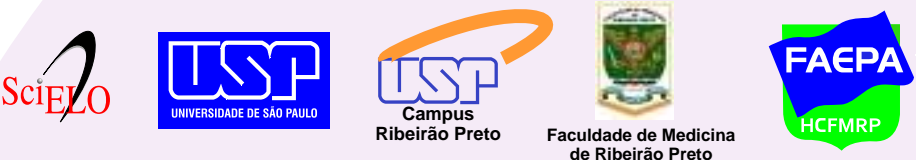

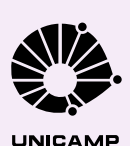

$\oplus$ SHIMADZU

GE Healthcare
Hotsite of proteomics metabolomics developped by:

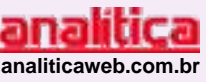

Thermo SCIENTIFIC 


\title{
Overexpression of kermit/dGIPC is associated with lethality in Drosophila melanogaster
}

\author{
G.B. Pereira1 , V. Valente ${ }^{1,2}$, M.S. de Queiroz ${ }^{1}$, M.C.B. Vianna ${ }^{1}$ and M.L. Paçó-Larson ${ }^{1}$ \\ ${ }^{1}$ Departamento de Biologia Celular e Molecular e Bioagentes Patogênicos, \\ 2Departamento de Cirurgia e Anatomia, Faculdade de Medicina de Ribeirão Preto, \\ Universidade de São Paulo, Ribeirão Preto, SP, Brasil
}

\begin{abstract}
Insertional mutagenesis is an important tool for functional genomics in Drosophila melanogaster. The insertion site in the KG00562 mutant fly line has been mapped to the CG8709 (herein named DmLpin) locus and to the 3' of kermit (also called $d G I P C)$. This mutant line presents a high lethality rate resulting from a gain of function. To obtain some insight into the biological role of the mutated locus, we have characterized the mutation and its relation to the high mortality of the KG00562 fly line. In this mutant, we did not detect one of the DmLpin transcripts, namely DmLpinK, but we did detect an unusual 2.3-kb mRNA (LpinK-w). Further investigation revealed that the LpinK-w transcript results from an aberrant splicing between the untranslated first exon of DmLpinK and the mini-white marker gene. Lack of DmLpinK or LpinK-w expression does not contribute to lethality, since heterozygous KG00562/Def7860 animals presented lethality rates comparable to those of the wild type. In contrast, the overexpression of kermit was associated with lethality of the KG00562 fly line. Significantly higher levels of kermit were detected in the Malpighian tubules of KG00562/+ flies that presented higher lethality rates than wild-type or KG00562/Def7860 animals, in which the lethality was rescued. In agreement with a recently reported study, our data support the hypothesis that misexpression of kermit/dGIPC could interfere with Drosophila development, with further investigations being needed in this direction.
\end{abstract}

Key words: Insertional mutagenesis; Aberrant splicing; DmLpin; Kermit/dGIPC

\section{Introduction}

The kermit gene was first described in Xenopus (1) and it was later shown that it shares $72 \%$ identity with mammalian GIPC proteins (2). The PDZ-domain of GIPC proteins has been identified in several organisms through its interaction with a variety of binding partners, including many membrane proteins (3). Despite several reports on Xkermit/GIPC proteins, their endogenous functions and the physiological roles of their interactions with several binding proteins are not well known. However, studies of loss of function in Xenopus have shown that kermit is required for frizzled-3 induction in the neural crest of ectodermal explants $(1,4)$ and for insulin-like growth factor (IGF) signaling during the development of Xenopus eye $(1,3)$. A recent report on Drosophila suggests that kermit (which was named dGIPC) might participate in the determination of the planar cell polarity of the wing epithelium by a mechanism independent of frizzled-3 (5). In addition, $d G I P C$ loss of function was reported to be associated with Drosophila motor activity and longevity (6).

The KG00562 mutant contains the insertion of a trans- poson, with a P element-based construction P(SUPor-P) (7) in the first intron of a new isoform of the CG8709, named DmLpinK (8), and in the 3' flanking region of the kermit gene. Interestingly, the untranslated first exon of kermit corresponds to the first exon of the DmLpinK isoform (Figure $1 \mathrm{~A})$. The isoforms encoded by $D m L$ pin are homologous of lipins (8), which constitute a novel family of $\mathrm{Mg}^{2+}$-dependent phosphatidate phosphatase (PAP1) enzymes (9). These enzymes catalyze the dephosphorylation of phosphatidic acid to yield diacylglycerol. Lipins play important roles in several cellular and physiological processes, as shown by studies performed on other organisms, including yeast, worms, and mammals (for a review, see Refs. 10,11). The yeast lipin homolog known as Pah1 p controls the phospholipid biosynthesis required for the nuclear and endoplasmic reticulum membranes $(12,13)$. The Caenorhabditis elegans lipin is also needed for the maintenance of normal nuclear and endoplasmic reticulum morphology $(14,15)$. In mammals, lipins are involved in the control of fatty acid metabo-

Correspondence: M.L. Paçó-Larson, Departamento de Biologia Celular e Molecular e Bioagentes Patogênicos, FMRP, USP, Av. Bandeirantes, 3900, 14049-900 Ribeirão Preto, SP, Brasil. Fax: +55-16-3633-1786. E-mail: mlplarso@fmrp.usp.br

Received October 14, 2010. Accepted February 4, 2011. Available online February 25, 2011. Published April 11, 2011. 
lism (16) and in adipocyte differentiation $(17,18)$. Data on Drosophila lipin function are virtually inexistent.

To gain some insights on the biological roles of $D m L$ pin and/or Kermit we have investigated the molecular bases of the semi-lethal mutation found in the KG00562 fly line. We show that the DmLpinK isoform is not detected in the KG00562 fly line, whereas an unusual $2.3 \mathrm{~kb}$ mRNA is expressed instead. We also observed that this mutant presents a high lethality rate that was not rescued in the wild-type background, characterizing a gain of function phenotype. Hence, we asked whether the lack of DmLpinK or the expression of this unusual mRNA was associated with this high lethality rate. We demonstrated that neither of these alterations is related to the lethality phenotype of the KG00562 fly line. Interestingly, we found that the overexpression of the kermit gene in Malpighian tubules seems to be associated with the gain of function observed in the KG00562 fly line.

\section{Material and Methods}

\section{Drosophila lines and culture}

The flies were grown in standard cornmeal/agar media at $25^{\circ} \mathrm{C}$. Cantons (CS) and $\mathrm{w}^{1118}$ flies were obtained from the laboratory of Dr. R.G.P. Ramos, FMRP, USP (Ribeirão Preto, SP, Brazil). The KG00562 [y $\mathrm{y}^{1} \mathrm{w}^{67 \mathrm{c} 23}$; P(SUPor-P) KG00562], and Def7860 (w ${ }^{1118 ;}$ Df(2R)Exel7095/CyO) fly lines were obtained from the Bloomington Stock Center (Bloomington, USA). The KG00562 mutant carries a $\mathrm{P}$ (PUPor-P) insertion in which the 3 ' has been mapped to the $4,033,487$ position on the $2 R$ chromosome $(7,19)$. The $\operatorname{Df}(2 \mathrm{R})$ Exel7095 has a $\sim 100 \mathrm{~kb}$ deletion that extends from position $4,012,164$ to position $4,119,968$ of the $2 \mathrm{R}$ chromosome.

RNA extraction, Northern blot, 3' end RACE cloning, and sequencing

Drosophila total RNA was extracted using Trizol reagent (Invitrogen, New Zealand) according to manufacturer instructions. PolyA $^{+}$RNA was purified with the OligoTex mRNA midi kit (Qiagen, Germany). In Northern blot hybridization experiments, RNA was fractionated on $1 \%$ agarose formaldehyde-denaturing gels and blotted to nylon membranes (Hybond N, Amershan, UK). Probe labeling, hybridization and post-hybridization washes were performed essentially as described by Sambrook et al. (20). The final washes were performed at $65^{\circ} \mathrm{C}$ in the presence of $0.1 \mathrm{XSSC}$ and $0.2 \%$ SDS. The cDNA of LpinB-w was cloned by rapid amplification of cDNA3'-end (RACE) using the 3'RACE System for Rapid Amplification of cDNA ends (Invitrogen, USA). A total of 500 ng KG00562 polyA ${ }^{+}$RNA in $20 \mu \mathrm{L}$ buffer containing $500 \mathrm{nM}$ of the adaptor primer was incubated at $70^{\circ} \mathrm{C}$ for $10 \mathrm{~min}$ and cooled on ice for $1 \mathrm{~min}$. The reaction proceeded for $15 \mathrm{~min}$ at $70^{\circ} \mathrm{C}$ in $20 \mathrm{mM}$ Tris- $\mathrm{HCl}, \mathrm{pH} 8,50 \mathrm{mM} \mathrm{KCl}, 10 \mathrm{mM}$ DTT, $0.5 \mathrm{mM}$ dNTP, and $200 \mathrm{U}$ Superscript II-RT (Invitrogen). PCR was performed with 3'-RACE-Ready cDNAs $(2 \mu \mathrm{L})$ in a $50-\mu \mathrm{L}$ reaction mixture containing $0.2 \mathrm{mM}$ dNTPs, 0.2 mM LpinB/Kermit first exon-specific primer (GSP-RACE-3'; Table 1), $0.2 \mathrm{mM}$ Abridged Universal Amplification Primer (AUAP), $20 \mathrm{mM}$ Tris- $\mathrm{HCl}, \mathrm{pH} 8.4,50 \mathrm{mM} \mathrm{KCl}, 1.5 \mathrm{mM}$ $\mathrm{MgCl}_{2}$, and $0.1 \cup$ Taq DNA polymerase (Fermentas, USA). The 3'-end was obtained with 37 cycles of denaturation $\left(94^{\circ} \mathrm{C}\right.$ for $\left.2 \mathrm{~min}\right)$, annealing $\left(66^{\circ} \mathrm{C}\right.$ for $\left.45 \mathrm{~s}\right)$, and extension $\left(72^{\circ} \mathrm{C}\right.$ for $\left.4 \mathrm{~min}\right)$. Finally, the $3^{\prime}-\mathrm{RACE}-\mathrm{PCR}$ product was gel-purified, cloned, and fully sequenced using the BigDye Terminator Cycle Sequencing Ready Reaction (PEApplied Biosystems, USA), M13 primers, and gene-specific primers (Table 1). Sequencing was performed with an ABI 3100 Genetic Analyzer (Applied Biosystems-HITACHI, USA). Sequence analysis and clustering were performed using the STADEN software package and the amino acid sequence alignment was done using ClustalW.

\section{Quantitative real-time RT-PCR}

For quantitative real-time RT-PCR (qRT-PCR), $700 \mathrm{ng}$

Table 1. Primer sequences used in the present study.

\begin{tabular}{|c|c|c|c|}
\hline Primer & Sequence $\left[5^{\prime} \rightarrow 3^{\prime}\right]$ & GenBank ID* & Amplicon size (bp) \\
\hline GSP-RACE-3' & GTCCGCTTTCCTCGCTCATTAG & NM_143771.2 & - \\
\hline W2 Exon-F & CGGTATGTGTCGCTCGTTG & NM_057439.2 & - \\
\hline W3 Exon-F & CGGCGGAGAAAGGAAGCGT & NM_057439.2 & - \\
\hline W3 Exon-R & ATGGCTTGGAAAATCAGGTGTT & NM_057439.2 & - \\
\hline W4 Exon-F & GCCATTAGCAAAGTAGCCCG & NM_057439.2 & - \\
\hline W4 Exon-R & TCCTTGAGCACCGACAGCC & NM_057439.2 & - \\
\hline W5 Exon-F & ACGCAAGTGGGTGTGATGAAT & NM_057439.2 & - \\
\hline W6 Exon-F & ACTGGTCTTCACGGCGATTG & NM_057439.2 & - \\
\hline W6 Exon-R & AGCAGACCCTCGTTGGCGT & NM_057439.2 & - \\
\hline \multirow[t]{2}{*}{ DmLpinK } & F - GCCACCACTATCATTATCAACA & GU327733 & 151 \\
\hline & R - TGGCGAAGGAATCGGCTCTA & & \\
\hline \multirow[t]{2}{*}{ Kermit } & F - GCCACCACTATCATTATCAACA & NM_143771.2 & 117 \\
\hline & R - GGACGGTTACCTTGCTCTTG & & \\
\hline \multirow[t]{2}{*}{ LpinK-w } & F - GCCACCACTATCATTATCAACA & HM000003 & 130 \\
\hline & R - CCGAAGCCCTGGTTAATGCA & & \\
\hline \multirow[t]{2}{*}{$R p L 32 A$} & F - GACCATCCGCCCAGCATAC & NM_079843.2 & 140 \\
\hline & R - CGCACTCTGTTGTCGATACC & & \\
\hline
\end{tabular}

${ }^{*}$ Accession number for the sequence used to determine each primer. 
RNA was converted to cDNA with SuperScript II (Invitrogen). To amplify and detect the isoform-specific PCR products we used the SYBR Green PCR Master Mix (PE Applied Biosystems) according to the manufacturer protocol. The primer sequences are shown in Table 1. Reactions without template were run in parallel for all plates to verify purity of measurements within each experiment. Each run was completed with a melting curve analysis to confirm the specificity of the amplification and to confirm absence of primer dimers. The mean threshold cycle of a mixture of equal amounts of CS and KG00562 RNA was used as a reference sample. The relative mRNA expression levels of target transcripts and the RpL32A gene (housekeeping) were quantified using a Gene Amp ${ }^{\circledR} 7500$ Sequence Detection System (PE Applied Biosystems). The qRT-PCR analyses were repeated three times and data were analyzed statistically by one-way analysis of variance (ANOVA) and by the Tukey multiple-comparison test (GraphPad Prism, version 5.0).

\section{Results}

\section{Characterization of the anomalous mRNA (LpinK-w) expressed in the KG00562 mutant fly}

Northern blot of mRNA from both heterozygous and homozygous KG00562 mutant flies probed with a fragment derived from the first exon of DmLpinK and kermit (probe B, Figure $1 \mathrm{~B}$ ) revealed that an mRNA of about $2.3 \mathrm{~kb}$ was not detected in CS wild-type flies. This unusual mRNA was not detected in the mutant when a DmLpinA-specific probe was used. Moreover, DmLpinK mRNA was not detected in the KG00562 flies, although the signal of kermit seemed to be

A

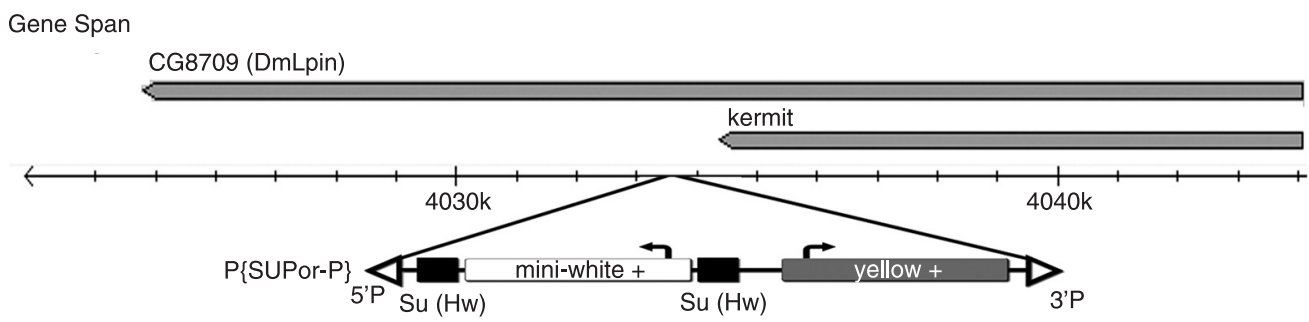

B
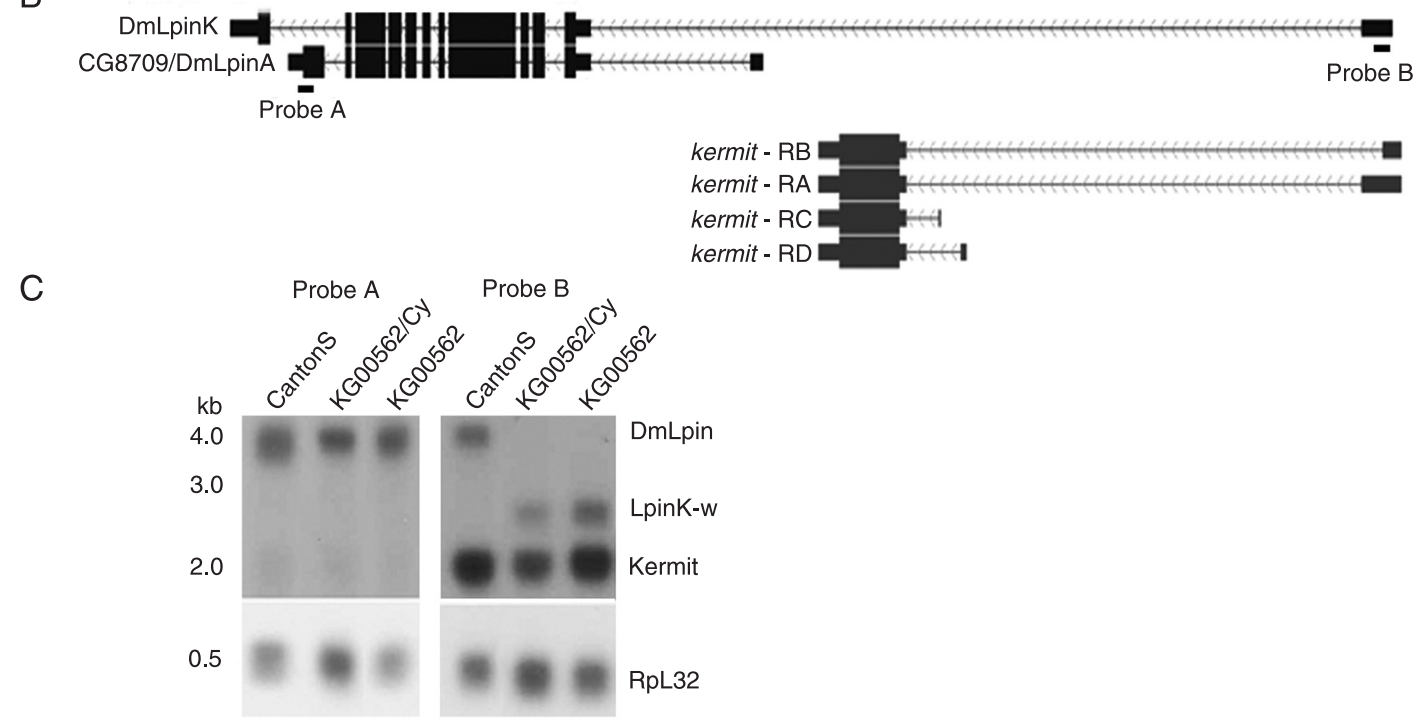

Figure 1. KG00562 expresses an anomalous mRNA detected by a probe for the $5^{\prime}$ UTR of DmLpinK and kermit. A, Map of CG8709 (DmLpin) and kermit gene span and the insertion site of the transposon P(SUPor-P) (7) in the genome of the KG00562 Drosophila fly line. B, Schematic representation of the exon-intron organization of DmLpinA (CG8709-RA) and DmLpinK (GU327733) and predicted isoforms A, B, C, and D of the kermit gene. The exons are represented by bars and the introns by lines. Noncoding sequences are shown as narrower portions of the exon bars. The arrowheads on the intron lines indicate the transcription direction. C, Northern blots of polyA ${ }^{+} \mathrm{RNA}_{\text {of }}$ CantonS, KG00562/Cy, and KG00562 flies probed with the last exon exclusive of DmLpinA (probe A) or with a fragment of the first exon of the DmLpinK and kermit (probe B). Note that probe B, but not probe A, detected an mRNA of about $2.5 \mathrm{~kb}$ (LpinB-w) in flies with the KG00562 background. The DmLpinK was not detected in lines carrying the insertion. 
unaffected (Figure 1C). To determine the primary structure of the KG00562-specific transcript we performed 3'-RACE $\mathrm{PCR}$ using a gene-specific primer based on the sequence of probe $\mathrm{B}$. The cloned cDNA revealed a sequence of 2305 bp (HM000003). The alignment of this sequence with the D. melanogaster genome showed that this mRNA contains part of the untranslated first exon of DmLpinK and kermit, and the second, third, fourth, fifth, and sixth exons of the white gene (Figure 2A). The presence of the DmLpinK/ kermit first exon acceptor site and the donor splicing site of the second exon of white indicate that this new mRNA resulted from an aberrant splicing between the DmLpinK

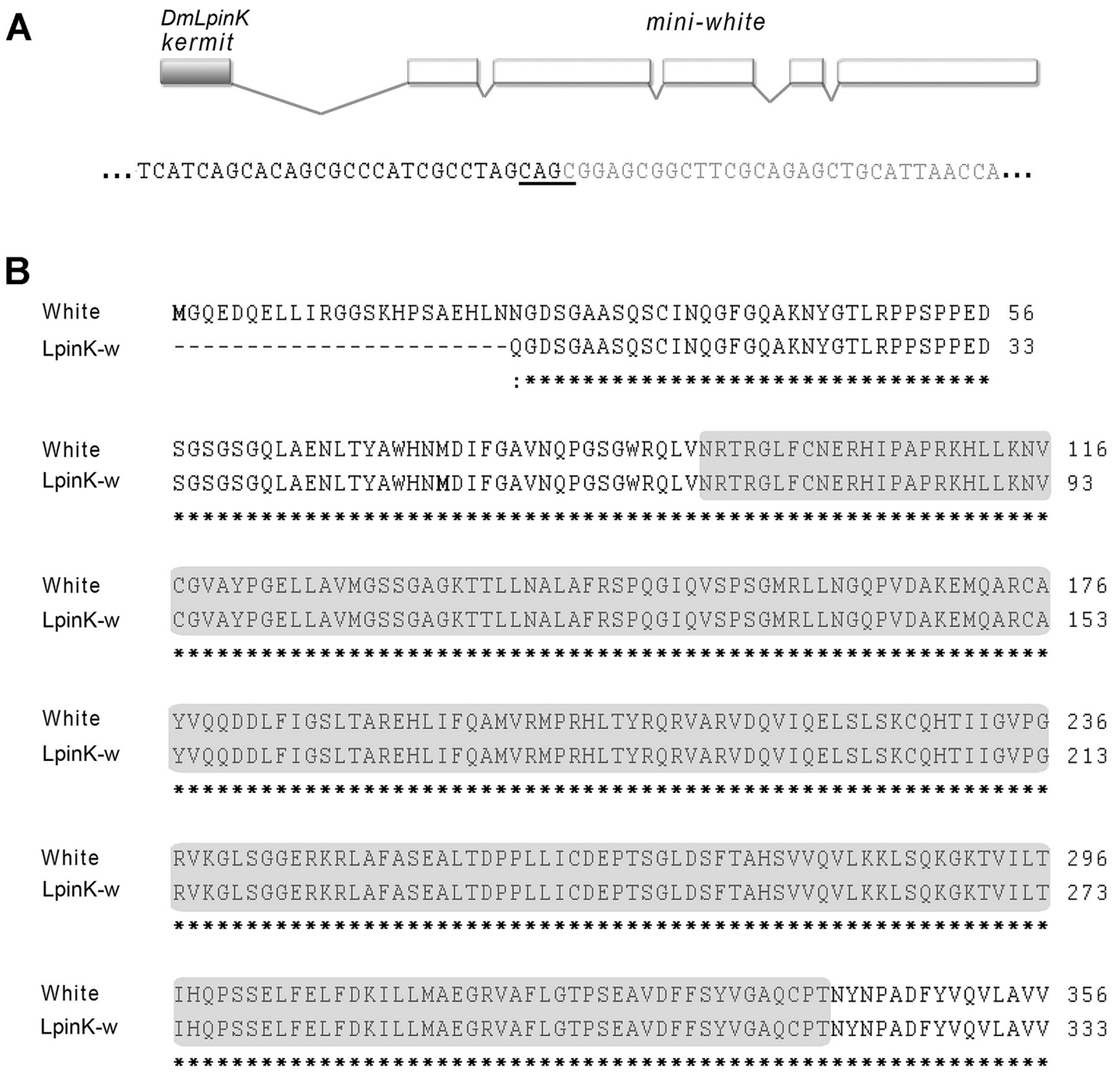

Figure 2. The anomalous mRNA expressed in the KG00562 fly is a chimera resulting from aberrant splicing. A, Diagram of the exonintron structure of the anomalous mRNA named LpinK-w. The first exon (grey box) corresponds to the 5'UTR of the DmLpinK and kermit and the other five boxes (white) correspond to the second, third, fourth, fifth, and sixth exons of the mini-white marker gene. The nucleotide sequence of the mRNA 5' region, including the first and second spliced exons, is shown below. The DmLpinK and kermit first exon 3' sequence is in black and the white second exon 5' sequence is in grey. The donor and acceptor splice sites are underlined. $B$, Alignment between amino acid sequences of the white protein and the polypeptide deduced from the LpinK-w mRNA. Note that the ABC domain (ATP Binding Cassette Transporter Complex) highlighted by the grey box is maintained in a polypeptide encoded by the chimeric mRNA LpinK-w. 
or kermit first exon and the second exon of the mini-white gene, present in the $\mathrm{P}(\mathrm{SUP}$ or- $\mathrm{P})$ transposon. The mRNA expressed in the KG00562 mutant was named LpinK-w (Figure 2A). The open reading frame of LpinK-w encodes a 770-amino acid sequence that corresponds to the white protein lacking the first 23 amino acids, which maintains the ATP Binding Cassette (ABC) Transporter Complex domain required for its activity (Figure $2 B$ ).

\section{Lethality of flies carrying the KG00562 insertion in different genetic backgrounds}

Mutant flies homozygous for the KG00562 insertion presented $48 \%$ lethality during development, which was significantly higher $(P<0.001)$ than the lethality observed for $w-\left(w^{1118}\right)$ or CS flies. The high lethality rate of KG00562 was not rescued in the progeny obtained from crosses of the mutant with $w^{1118}$ or CS flies, which presented an average lethality of 52 and $60 \%$, respectively (Table 2). These results suggest that the lethality associated with the KG00562 fly line is the result of a gain of function mutation. Additionally, the lethality of the progeny obtained from crosses of the KG00562 line with flies carrying a 100-kb deletion (Def7860), which covers the mutated region, did not differ from the lethality of CS wild-type flies (Table 2), indicating that the gain of function phenotype observed in KG00562 flies is related to alterations in this genomic region.

\section{Tissue expression of DmLpinK, kermit, and LpinK-w} in animals with different genetic backgrounds

To determine whether the chimeric mRNA LpinK-w expressed in the KG00562 fly line results from an aberrant splicing of DmLpinK or kermit mRNAs, we compared the expression profile of LpinK-w with that of DmLpinK and kermit transcripts in the KG00562 mutant and wild-type tissues. These analyses were performed with total RNA extracted from the central nervous system (CNS), intestinal tract, and Malpighian tubules of 3rd-instar larvae, because of previous indications that $D m L$ pin isoforms were differentially expressed in these tissues (8).

Confirming our initial data, the DmLpinK transcripts were expressed in significantly higher levels $(P<0.001)$ in the CNS of wild-type animals than in the other tissues analyzed, but were not detected in the tissues of the KG00562 mutant (Figure 3). The chimeric LpinK-w presented an expression

Table 2. Lethality of the progenies from crosses between different fly lines.

\begin{tabular}{lcc}
\hline Cross $(\varphi \times ð)$ & Total number of eggs & Lethality $(\%)$ \\
\hline CS $\times$ CS & 633 & $19 \pm 3$ \\
w $^{1118} \times$ w $^{1118}$ & 1030 & $20 \pm 9$ \\
KG00562 x KG00562 & 602 & $48 \pm 2^{*}$ \\
KG00562 x CS & 817 & $60 \pm 7^{*}$ \\
KG00562 x w 1118 & 872 & $52 \pm 8^{*}$ \\
CS $\times$ Def7860/Bc & 492 & $17 \pm 4$ \\
KG00562 x Def7860/Bc & 587 & $16 \pm 3$ \\
\hline
\end{tabular}

Data are reported as means \pm SEM. * $P<0.001$ compared to wild-type (CantonS, CS), w- $\left(w^{1118}\right)$ and KG00562/Def7860 flies (Tukey test).

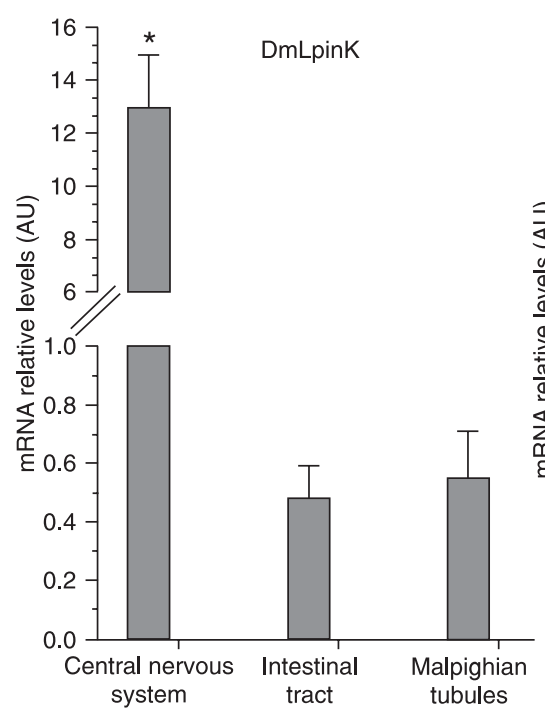

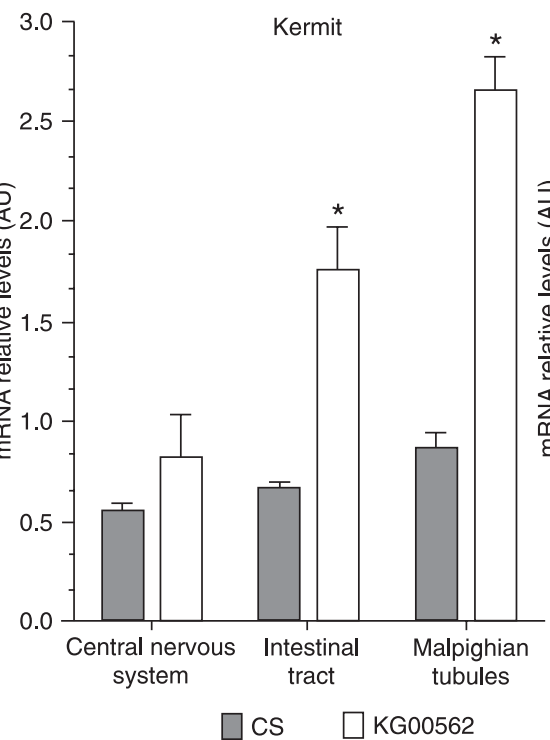

CS

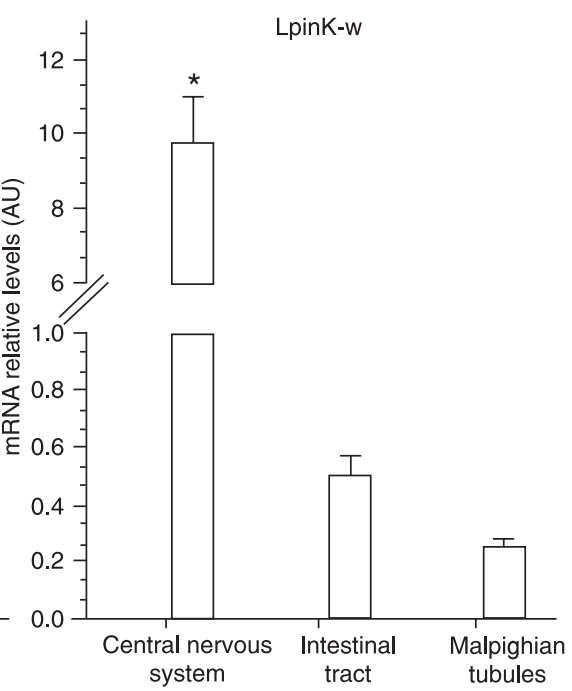

system

Figure 3. The expression of DmLpinK and kermit is affected in the KG00562 line. qRT-PCR of DmLpinK, kermit, and LpinK-w are shown in different tissues (central nervous system, intestinal tract, and Malpighian tubules) of the KG00562 mutant and CantonS (wild type) 3rd-instar larvae. Data are reported as means \pm SEM $(\mathrm{N}=3)$. For DmLpinK and LpinK-w, the asterisks denote significant differences between tissues ( ${ }^{*} P<0.001$, Tukey test). In the case of Kermit, the comparison was between CS and KG00562 ("$P<0.001$, Tukey test). 

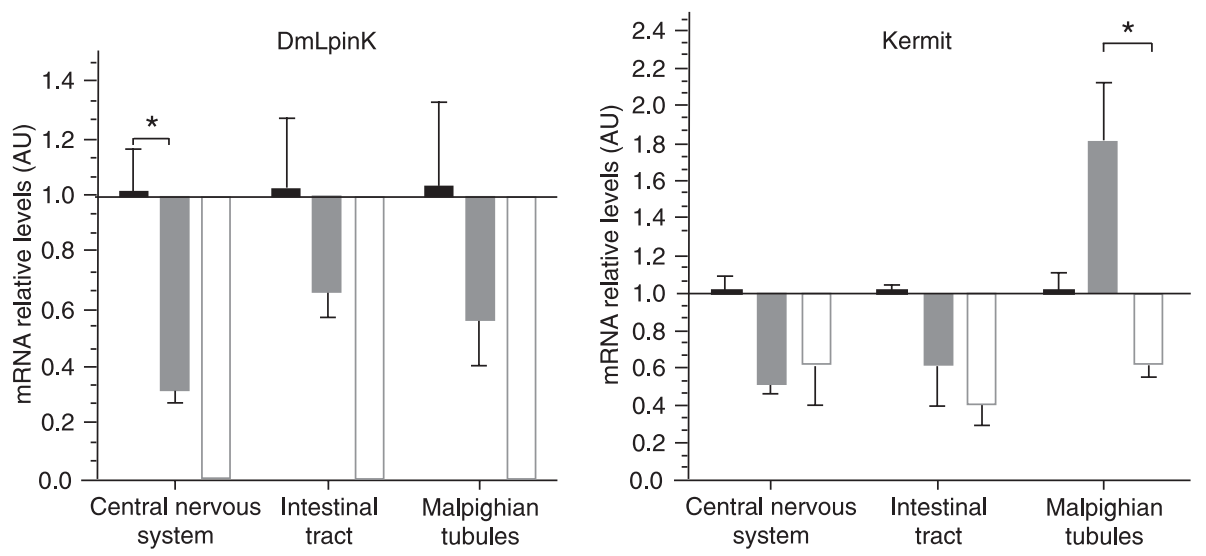

Figure 4. Overexpression of kermit in the Malpighian tubules is maintained in animals carrying KG00562 mutation in CantonS wild-type background (CS). qRT-PCR of DmLpinK and kermit in different tissues (central nervous system, intestinal tract, and Malpighian tubules) of 3rd-instar larvae. The tissues analyzed were from the progenies of crosses between KG00562 x CantonS (CS) and KG00562 x Def7860. Data are reported as means \pm SEM $(N=3)$ of each mRNA level in comparison to its level in CS. Asterisks denote significant differences between groups ( ${ }^{*}<0.001$, Tukey test).

pattern virtually identical to that of the wild-type DmLpinK mRNA (Figure 3). Kermit transcripts were detected at low levels in all tissues of the wild-type CS larvae, while in the KG00562 mutant their levels were significantly higher $(\mathrm{P}<$ $0.001)$ in the intestinal tract and in the Malpighian tubules when compared to the wild type (Figure 3).

The reduction of DmLpinK and/or the overexpression of kermit in the different larval tissues could be associated with the lethality of KG00562 flies. To address this question we measured the levels of these mRNAs in the progeny of crosses between KG00562 and CS or Def7860 animals, where the lethality was approximately 60 and $17 \%$, respectively (Table 2). DmLpinK levels were reduced in all tissues analyzed of animals carrying the KG00562 insertion in both genetic backgrounds when compared to the wild-type flies (Figure 4). Similarly, kermit levels were also reduced in the CNS and intestinal tract of larvae carrying the KG00562 insertion in both genetic backgrounds. Conversely, in the Malpighian tubules, the amounts of kermit transcripts in the KG00562/+ and KG00562/Def7860 flies differed significantly, being increased in the former ( 1.8-fold) and reduced in the latter ( $\sim 0.6$-fold) progenies compared to wild-type animals (Figure 4).

\section{Discussion}

In this study we investigated the molecular bases of the semi-lethal mutation found in the KG00562 fly line. Our data show that the insertion of the P(SUPor-P) transposable element in KG00562 resulted in an aberrant splicing between DmLpinK and the mini-white marker gene (Figure 2). A similar mutagenic event has been reported for the $P Z$ lacZ rosy transposon. The insertion of this transposon in the second intron of the pipsqueak gene resulted in an aberrant splicing and in events of premature transcription termination that produced an mRNA composed of 5' sequences from pipsqueak and 3' sequences from the PZ element (21). Such a mutation could yield chimeric proteins with dominant effects. However, this is not the case for KG00562. We observed that, rather than a chimeric protein, the aberrant mRNA(LpinK-w) expressed in the KG00562 fly line encodes an aminoterminal deleted white protein containing the $A B C$ domain (ATP Binding Cassette; Figure 2). The ABC domain is required for the white function in transporting molecules across membranes, such as the pigment precursors tryptophan and guanine (22-24), and the cGMP in Malpighian tubules (25). Lack of the white function impairs pigment production in the light-screening cells of the compound eye resulting in flies with white eyes, which is the most prominent phenotype of $w$ mutants. The eye color provided by the miniwhite expression in a $w$ - $\left(w^{67 c 23}\right)$ recipient line was used to identify P(SUPor-P) transformants (5); thus the LpinK-w expression in the KG00562 fly line does not interfere with the white function. Ectopic overexpression of white, driven by a heat shock promoter, led to a marked change in the sexual behavior of mature adult males (26). Therefore, misexpression of the truncated white could contribute to the lethality of the KG00562 line. However, significantly higher rates of lethality $(P<0.001)$ were still observed in heterozygous KG00562/w+ or KG00562/w- flies (Table 2), while in heterozygous animals carrying the KG00562 insertion over a deletion that covers the mutated region (Table 2) the lethality phenotype was rescued. These data suggest that, rather than the expression of the chimeric LpinK-w mRNA, alterations in other genes covered by this deletion (Def7860) might be related to the lethality. Lack of DmLpinK 
expression does not seem to contribute to lethality, since heterozygous KG00562/Def7860 animals, where DmLpinK expression does not occur (Figure 4), present lethality rates similar to those of the CS wild type (Table 2). It is possible that the expression of the DmLpinA isoform, which seems to be normally expressed in the KG00562 fly line (Figure 1 ), compensates for the lack of the DmLpinK isoform. Accordingly, recent data showed that the increase of lipin-2 levels counterbalance the PAP1 activity in the liver of mice deficient in Lpin1 (27).

The overexpression of kermit was revealed to be associated with the lethality of the KG00562 fly, although we cannot rule out the involvement of other genes covered by the Def7860 deficiency. Significantly higher levels of kermit (1.8- to 3.0-fold) were observed in the Malpighian tubules of KG00562/+ flies in comparison to both CS line and KG00562/Def7860 animals (Figure 4). Since in the heterozygous KG00562/+ flies the lethality is highly elevated and in heterozygous KG00562/Def7860 the lethality phenotype is rescued to levels similar to those of CS wild type (Table 2), the overexpression of kermit is the only alteration observed that can be associated with the KG00562 lethality. This agrees with a recent report showing that,

\section{References}

1. Tan C, Deardorff MA, Saint-Jeannet JP, Yang J, Arzoumanian A, Klein PS. Kermit, a frizzled interacting protein, regulates frizzled 3 signaling in neural crest development. Development 2001; 128: 3665-3674.

2. De Vries L, Lou X, Zhao G, Zheng B, Farquhar MG. GIPC, a PDZ domain containing protein, interacts specifically with the $C$ terminus of RGS-GAIP. Proc Natl Acad Sci U S A 1998; 95: $12340-12345$.

3. Wu J, O'Donnell M, Gitler AD, Klein PS. Kermit 2/XGIPC, an IGF1 receptor interacting protein, is required for IGF signaling in Xenopus eye development. Development 2006; 133: 3651-3660.

4. Rasmussen JT, Deardorff MA, Tan C, Rao MS, Klein PS, Vetter ML. Regulation of eye development by frizzled signaling in Xenopus. Proc Natl Acad Sci U S A 2001; 98: 38613866.

5. Djiane A, Mlodzik M. The Drosophila GIPC homologue can modulate myosin based processes and planar cell polarity but is not essential for development. PLoS One 2010; 5: e11228.

6. Kim J, Lee S, Ko S, Kim-Ha J. dGIPC is required for the locomotive activity and longevity in Drosophila. Biochem Biophys Res Commun 2010; 402: 565-570.

7. Roseman RR, Johnson EA, Rodesch CK, Bjerke M, Nagoshi RN, Geyer PK. A P element containing suppressor of hairywing binding regions has novel properties for mutagenesis in Drosophila melanogaster. Genetics 1995; 141: 1061-1074.

8. Valente V, Maia RM, Vianna MC, Paco-Larson ML. Drosophila melanogaster lipins are tissue-regulated and developmentally regulated and present specific subcellular distributions. FEBS J 2010; 277: 4775-4788. although kermit/dGIPC is not essential for development, increased doses of this gene can be deleterious. It was demonstrated that overexpression of $d G P I C$ on the dorsal thorax interferes with the generation of planar cell polarity in the wing (5). Further investigations on the effect of kermit/ $d G I P C$ overexpression in specific tissues or developmental stages should contribute to understanding the role of this gene in Drosophila development.

\section{Acknowledgments}

The KG00562 and Def7860 lines were provided by the Bloomington Stock Center at Indiana University (Bloomington, IN, USA). We thank Cirlei A.V. Saraiva and Benedita O. Souza for dedicated technical assistance, and Tania Paula Aquino Defina for DNA sequencing assistance. Research supported by FAPESP (\#2007/50173-8), CNPq (\#479444/2008-0) and stipends from FAEPA-FMRP. G.B. Pereira (\#07/52871-4), V. Valente (\#01/07696-3), and M.S. de Queiroz (\#07/52870-8) were supported by fellowships from FAPESP, and M.C.B. Vianna received a fellowship from CAPES. M.L. Paçó-Larson is the recipient of a Research Productivity fellowship from CNPq (\#303197/2009-9).
9. Han GS, Wu WI, Carman GM. The Saccharomyces cerevisiae Lipin homolog is a $\mathrm{Mg}^{2+}$-dependent phosphatidate phosphatase enzyme. J Biol Chem 2006; 281: 9210-9218.

10. Siniossoglou S. Lipins, lipids and nuclear envelope structure. Traffic 2009; 10: 1181-1187.

11. Reue K. The lipin family: mutations and metabolism. Curr Opin Lipidol 2009; 20: 165-170.

12. Santos-Rosa H, Leung J, Grimsey N, Peak-Chew S, Siniossoglou S. The yeast lipin Smp2 couples phospholipid biosynthesis to nuclear membrane growth. EMBO J 2005; 24: 1931-1941.

13. O'Hara L, Han GS, Peak-Chew S, Grimsey N, Carman GM, Siniossoglou S. Control of phospholipid synthesis by phosphorylation of the yeast lipin Pah1p/Smp2p Mg ${ }^{2+}$-dependent phosphatidate phosphatase. J Biol Chem 2006; 281: 3453734548.

14. Golden A, Liu J, Cohen-Fix O. Inactivation of the C. elegans lipin homolog leads to ER disorganization and to defects in the breakdown and reassembly of the nuclear envelope. $J$ Cell Sci 2009; 122: 1970-1978.

15. Gorjanacz M, Mattaj IW. Lipin is required for efficient breakdown of the nuclear envelope in Caenorhabditis elegans. $J$ Cell Sci 2009; 122: 1963-1969.

16. Reue K, Zhang P. The lipin protein family: dual roles in lipid biosynthesis and gene expression. FEBS Lett 2008; 582: 90-96.

17. Phan J, Peterfy M, Reue K. Lipin expression preceding peroxisome proliferator-activated receptor-gamma is critical for adipogenesis in vivo and in vitro. J Biol Chem 2004; 279: 29558-29564.

18. Peterfy M, Phan J, Reue K. Alternatively spliced lipin iso- 
forms exhibit distinct expression pattern, subcellular localization, and role in adipogenesis. J Biol Chem 2005; 280: 32883-32889.

19. Bellen HJ, Levis RW, Liao G, He Y, Carlson JW, Tsang G, et al. The BDGP gene disruption project: single transposon insertions associated with $40 \%$ of Drosophila genes. Genetics 2004; 167: 761-781.

20. Sambrook J, Fitsch EF, Maniatis T. Molecular cloning: $A$ laboratory manual. Cold Spring Harbor: Cold Spring Harbor Press; 1989.

21. Horowitz $\mathrm{H}$, Berg $\mathrm{CA}$. Aberrant splicing and transcription termination caused by $\mathrm{P}$ element insertion into the intron of a Drosophila gene. Genetics 1995; 139: 327-335.

22. Sullivan DT, Bell LA, Paton DR, Sullivan MC. Genetic and functional analysis of tryptophan transport in Malpighian tubules of Drosophila. Biochem Genet 1980; 18: 1109-1130.

23. Summers KM, Howells AJ, Pyliotis NA. Biology of eye pig- mentation in insects. Adv Insect Phys 1982; 16: 119-166.

24. Shulenin S, Schriml LM, Remaley AT, Fojo S, Brewer B, Allikmets R, et al. An ATP-binding cassette gene (ABCG5) from the ABCG (White) gene subfamily maps to human chromosome 2p21 in the region of the Sitosterolemia locus. Cytogenet Cell Genet 2001; 92: 204-208.

25. Evans JM, Day JP, Cabrero P, Dow JA, Davies SA. A new role for a classical gene: white transports cyclic GMP. J Exp Biol 2008; 211: 890-899.

26. Zhang SD, Odenwald WF. Misexpression of the white (w) gene triggers male-male courtship in Drosophila. Proc Natl Acad Sci U S A 1995; 92: 5525-5529.

27. Gropler MC, Harris TE, Hall AM, Wolins NE, Gross RW, Han $\mathrm{X}$, et al. Lipin 2 is a liver-enriched phosphatidate phosphohydrolase enzyme that is dynamically regulated by fasting and obesity in mice. J Biol Chem 2009; 284: 6763-6772. 